\title{
基于硅酸镓镧的声表面波高温压力器件的微结构 加工制造技术研究*
}

\author{
梁晓瑞 ${ }^{1,2}$ 徐芳萌 ${ }^{1,2}$ 薛 涛 ${ }^{1,2}$ 谭秋林 ${ }^{1,2}$ 董和䂞 ${ }^{1,2}$ 熊继军 1,2 \\ (1. 中北大学电子测试技术国防重点实验室 太原 030051; \\ 2. 中北大学仪器科学与动态测试教育部重点实验室 太原 030051)
}

\begin{abstract}
摘要: 采用硅酸镓镧(Langasite, LGS)压电材料制备声表面波(Surface acoustic wave, SAW)高温压力传感器可解决高温、高压 等恶劣环境下温度、压力等参数的实时原位测试的问题, 但由于硅酸镓镧是一种高硬度难加工的硬脆性压电材料, 因此开展 了硅酸镓镧压电材料在高温等恶劣环境下的微结构加工制造技术的研究。通过分析 LGS 的化学与物理特性, 利用湿法腐蚀工 艺实现 LGS 的微纳成形制造和控制; 以及利用高温热压直接键合工艺实现密封空腔的制造, 研制出一套适用于 LGS 等硬脆 性材料的微纳结构成形制造技术, 并探究了微结构加工制造工艺的实用性。利用微结构加工制造技术实现的压力敏感微结构 制备了基于 LGS 的 SAW 高温压力传感器样机并进行了测试, 样机在 $25 \sim 1000{ }^{\circ} \mathrm{C} 、 20 \sim 500 \mathrm{kPa}$ 环境下能稳定工作, 验证 了 LGS 微纳结构成形制造技术在高温等恶劣环境下应用的可行性。
\end{abstract}

关键词: 硅酸镓镧; 声表面波; 湿法刻蚀; 热压键合; 高温压力

中图分类号: TG156

\section{Microstructure Manufacturing Technology of Acoustic Surface Wave High Temperature Pressure Devices Based on Langasite}

\author{
LIANG Xiaorui $^{1,2}$ XU Fangmeng ${ }^{1,2}$ XUE Tao ${ }^{1,2}$ TAN Qiulin ${ }^{1,2}$ \\ DONG Helei $^{1,2}$ XIONG Jijun ${ }^{1,2}$ \\ (1. National Defense Key Laboratory of Science and Technology on Electronic Test and Measurement, \\ North University of China, Taiyuan 030051; \\ 2. Key Laboratory of Instrumentation Science and Dynamic Measurement, Ministry of Education, \\ North University of China, Taiyuan 030051)
}

\begin{abstract}
A surface acoustic wave pressure sensor based on Langasite(LGS) can solve the problem of real-time in-situ measurement of temperature, pressure, and other parameters in harsh environments such as high temperature and high pressure. However, as the Langasite is a kind of hard and brittle piezoelectric material with high hardness and difficult to machine, the research on fabrication technology of microstructure of Langasite piezoelectric material in harsh environment such as high temperature is carried out. By analyzing the chemical and physical properties of LGS, the wet etching process is used to realize micro-nano fabrication and control of LGS. A sealed cavity microstructure is realized by high temperature hot pressing direct bonding process. A set of micro nanostructure forming technology suitable for LGS hard and brittle materials are developed, and the practicability of the microstructure processing and manufacturing process is explored. The SAW high-temperature pressure sensor prototype based on LGS is fabricated and tested by using pressure-sensitive microstructure realized by microstructure machining technology. The prototype can work stably at $25-1000{ }^{\circ} \mathrm{C}, 20-500 \mathrm{kPa}$, which verifies the feasibility of LGS microstructure forming technology in other harsh environments.
\end{abstract}

Key words: Langasite; surface acoustic wave; wet etching; thermocompression bonding; high temperature and pressure

* 国家自然科学基金航天联合基金重点资助项目(U1837209)。20201201 收 到初稿, 20210322 收到修改稿 


\section{0 前言}

在航空航天领域, 高超音速飞行器及其发动机 等装备, 表面由于气动加热以及燃烧产生超高温环 境 ${ }^{[1]}$, 局部温度甚至超过 $800{ }^{\circ} \mathrm{C}$, 工作压力超过 $1.2 \mathrm{MPa}$, 温度以及压力参数的实时监测对材料选型 以及失效分析具有重要意义 ${ }^{[2-3]}$ 。目前研究中尚无有 效手段可用于 $800{ }^{\circ} \mathrm{C}$ 以上高温环境下各参数的实 时原位获取。恶劣环境下温度、压力等参数的原位 实时获取, 对于航天飞行器的材料选型、结构设计 以及防护措施等具有重要意义 ${ }^{[4-6]}$, 因此迫切需要开 发出新型特种传感器, 实现恶劣环境下温度、压力 等参数的实时原位精确测试。恶劣环境下需选择具 有良好的高温稳定性、高压稳定性材料用于制备高 温、压力器件。随着 MEMS 技术的发展, 由于硅基 传感器具有小型化及高度集成性, 其广泛应用于实 际工程测试。然而, 传统硅基压力传感器在超过 $150{ }^{\circ} \mathrm{C}$ 环境下, 由于热本征态激发导致电隔离停止, 导致反向 PN 结失去作用、焊点丢失、欧姆接触失 效等问题, 最终导致传感器失效 ${ }^{[7-12]}$ 。针对以上问 题, 绝缘体上硅 $(\mathrm{SOI})$ 材料可以利用氧化硅介质隔离 替代硅基传感器中的 P-N 结隔离因此可以解决反向 PN 结损坏的问题, 但硅材料短时间最高温度只能达 到 $600{ }^{\circ} \mathrm{C}^{[13-17]}$ 。与硅材料相比, 碳化硅 $(\mathrm{SiC})$ 能够在 更高的温度下工作, 以及 $\mathrm{SiC}$ 由于材料本身超过 $500{ }^{\circ} \mathrm{C}$ 会发生塑性变形, 由于高温环境下 $\mathrm{SiC}$ 薄膜 压阻效应的退化, 无法在超过 $800{ }^{\circ} \mathrm{C}$ 环境下长时间 工作 ${ }^{[18-21]}$ 。可知传统的硅基测试技术难以实现高温、 高压等恶劣环境中各参数的准确实时监测。因此, 函需开发基于新型耐高温材料制备新型结构的传感 器以满足航空航天领域测试需求。与传统的有线有 源器件相比, 声表面波技术可实现远距离传感器信 号无线无源监测, 且声表面波传感器结构简单、体 积小、抗干扰性能强、稳定性好、可进行多参数的 无线无源的测试 ${ }^{[22-23]}$, 可满足在恶劣环境下无线测 试的需求。由于硅酸镓镧(Langasite, LGS)材料具有 较高熔点, 良好的力学特性, 因此其可作为耐高温 基底材料的良好候选材料。硅酸镓镧晶体是一种橙 色透明的新型压电晶体，机电耦合系数比石英高 $2 \sim 3$ 倍, 具有较高熔点 $\left(1470{ }^{\circ} \mathrm{C}\right)$, 从室温到熔点 间无相变, 且因其低声速、零温度切向和良好的高 温稳定性等优良性能成为制备声表面波(Surface acoustic wave, SAW) 高温压力传感器的理想材 料 ${ }^{[24-28]}$, 但 LGS 属硬脆性材料, 难加工和控制。为
了解决 LGS 材料难加工问题, 本文针对材料的高 温、化学等特性，采用湿法刻蚀的方式实现晶片表 面腔体结构微纳制造, 探究了晶片表面活化、高温 热压键合的微加工工艺, 实现硬脆性材料的晶片直 接键合，避免了因材料之间热应力失配导致的敏感 结构失效问题。试验利用刻蚀、键合、表面图形化 等微结构加工工艺设计并制造了声表面波高温压力 微传感器, 以及利用温压复合测试系统完成了传感 器在 $25 \sim 1000{ }^{\circ} \mathrm{C}, 20 \sim 500 \mathrm{kPa}$ 的环境下测试, 测 试结果表明传感器可在该条件下稳定工作, 验证了 硬脆性材料采用湿法刻蚀与热压键合工艺制造的膜 片式压力传感器在高温恶劣环境的工作能力。

\section{1 高温压力器件的微结构成形机理}

\section{1 刻蚀机理}

在硅酸镓镧耐高温材料上实现晶片表面图形化 仍是微加工领域的一个难题, 在微加工中采用刻蚀 技术实现晶片的图形化，且在晶片表面实现刻蚀技 术是 MEMS 微加工工艺和器件制造的关键步骤。刻 蚀技术是采用化学或者物理的方法有选择性的从晶 片表面去除不需要的材料的过程 ${ }^{[29]}$ 。在 MEMS 微加 工工艺中最常用的刻蚀技术为干法刻蚀和湿法刻 蚀。干法刻蚀是物理反应过程, 是用等离子体进行 薄膜刻蚀的技术 ${ }^{[30]}$ 。湿法刻蚀是化学反应过程, 利 用溶液和预刻蚀材料之间的化学反应来去除未被 掩蔽膜材料掩蔽的部分而达到刻蚀目的 ${ }^{[31]}$ 。根据 不同材料的不同化学或物理性质，选用合适的刻 蚀方法实现表面图形化。干法腐蚀的方法实现表 面图形化需要选择性足够高的腐蚀剂, 这种腐蚀 剂难实现, 且腐蚀速率较慢; 而硅酸镓锞材料易溶 于酸碱溶液, 易实现材料的表面图形化, 且常规的 硅酸钾镧较厚, 需要寻找较快的刻蚀速度形成较薄的 敏感膜片, 因此本文采用湿法刻蚀技术。试验采用不 同配比的酸溶液进行刻蚀，经试验验证，盐酸与磷酸 溶液 $1: 1$ 混合可实现较好的硅酸镓镧表面图形。 硅酸镓镧首先与盐酸反应, 酸中的 $\mathrm{H}^{+}$与硅酸镓锞 反应方程式如式(1)所示

$\mathrm{La}_{3} \mathrm{Ga}_{5} \mathrm{SiO}_{14}+\mathrm{HCl}+\mathrm{H}_{2} \rightarrow \mathrm{SiO}_{2}+\left(\mathrm{LaCl}_{3}+\mathrm{H}_{2} \mathrm{O}\right)+\mathrm{GaCl}_{3}$

$\mathrm{LaCl}_{3}$ 和 $\mathrm{GaCl}_{3}$ 溶解在刻蚀液中。采用热浓磷酸 去除二氧化硅。浓磷酸与二氧化硅反应的机理是熵 减。在加热条件下，硅酸与磷酸失去水分子，形成 杂多酸, 反应方程式如式(2)所示

$$
\mathrm{SiO}_{2}+\mathrm{H}_{3} \mathrm{PO}_{4} \rightarrow \mathrm{H}_{2} \mathrm{SiO}_{3}+\mathrm{H}_{6} \mathrm{P}_{4} \mathrm{O}_{13}+\mathrm{H}_{2} \mathrm{O}
$$


硅酸镓镧晶片刻蚀原理图如图 1 所示。通过控 制酸溶液的浓度以及反应温度, 实现刻蚀过程的精 确控制。

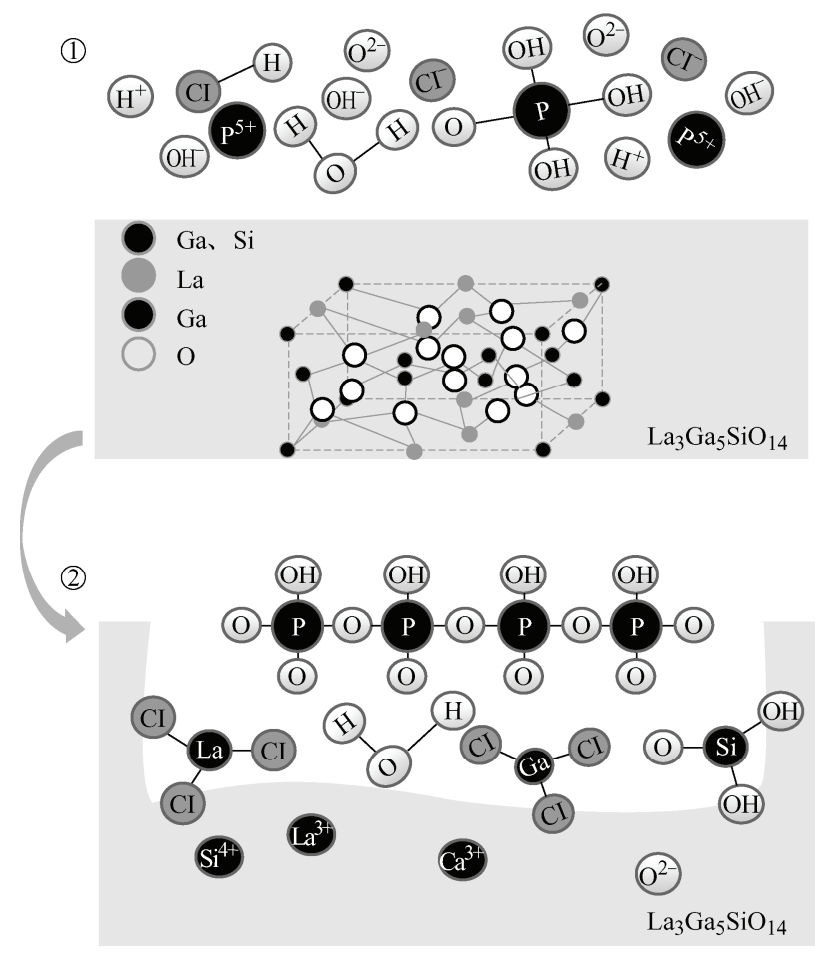

图 1 刻蚀原理图

\section{2 预键合机理}

在硅酸镓镧晶片实现热压键合之前首先要对基 底表面进行预键合, 表面预键合主要包括表面清洗 处理与表面活化处理等步骤。表面预键合经过活化 处理后, 通过两晶片表面分子及原子间的作用力相 互吸引, 形成自发键合的过程, 进而实现晶片之间 的结合 ${ }^{[32]}$ 。等离子体表面活化处理的目的是为了使 晶片表面获得悬挂键亲水性 $\mathrm{OH}^{-}$基团, 改变晶片表 面的化学状态, 增加晶片表面的活化能。亲水性处 理是使用活化后的含有着基的溶液对硅酸镓针晶体 表面进行处理。晶片表面活化处理利用 $\mathrm{O}_{2} 、 \mathrm{~N}_{2} 、 \mathrm{Ar}$ 等离子体处理实现, 在外加电场的作用下利用高能 粒子高速撞击晶圆表面, 达到去除晶片表面
杂质的目的，且使得晶片表面上原来不稳定的非桥 接原子获得能量后离开成键原子, 使表面获得大量 不饱和悬挂键。将处理后的晶片面放置在空气中时, 晶片表面吸附大量 $\mathrm{OH}^{-}$基团形成超亲水表面。将活 化处理后的硅酸镓锞晶片放入乙醇溶液中, 经处理 后, 在晶片表面产生高浓度的 $\mathrm{OH}^{-}$基团, 将两晶片 在室温下接触, 并在晶片表面施加力的作用, 两晶 片之间通过单层水分子和 $\mathrm{OH}^{-}$基团之间的氢键形成 微弱的分子间作用力从而达到预键合的效果。

\section{3 热压键合机理}

LGS 密封空腔的形成是 SAW 传感器应用在高 温、高压等恶劣环境下亟需解决的一个关键性难题。 高温压力传感器实现密封压力空腔需要将两个同种 或不同种材料之间进行键合, 但在高于 $500{ }^{\circ} \mathrm{C}$ 高温 环境中，不同材料的热膨胀系数不同，会造成传感 器结构失效。直接键合是指将同种材料的表面带有 刻蚀空腔与表面平整的晶片经预键合后, 在高温压 力的条件下实现两个晶片表面紧密接触, 最终实现 两晶片的密封粘合，避免了不同材料的热应力失配问 题。因此直接键合是解决热应力不匹配问题最为有效 的手段之一。直接键合经过高温处理获得键合所需能 量, 主要通过化学键之间的键能结合。经过活化处理 的晶片表面吸附了大量的亲水性 $\mathrm{OH}^{-}$基团, 当两晶片 在空气中直接键合时, 晶片表面的 $\mathrm{OH}^{-}$基团结合形成 水分子层, 当给预键合后的晶片施压加温时, 水分子 层在高温下蒸发, 使两晶片获得更高的键合强度。 由于分子的热运动和物体的温度有关, 物体的温度越 高, 分子的运动越快 ${ }^{[33]}$ 。两晶片经过 $\left(\geqslant 1000{ }^{\circ} \mathrm{C}\right)$ 高 温条件, 晶片内部分子在高温作用下分子运动加快, 同时给晶片施加压力, 打破了晶格的束缚, 使单位 体积内分子数量增多, 分子间隔减小, 两个键合面 的原子 $(\mathrm{Si} 、 \mathrm{La} 、 \mathrm{Ga} 、 \mathrm{O})$ 打破了晶格的束缚而发生换 位和移动, 产生了新的极键将两个表面的元素连接 起来, 发生了原子扩散, 从而获得密闭性良好的 LGS 压力空腔。热压键合原子扩散图如图 2 所示。

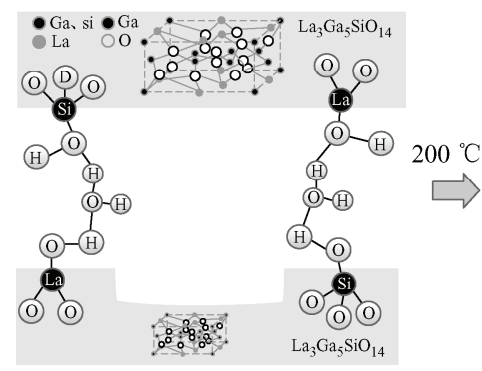

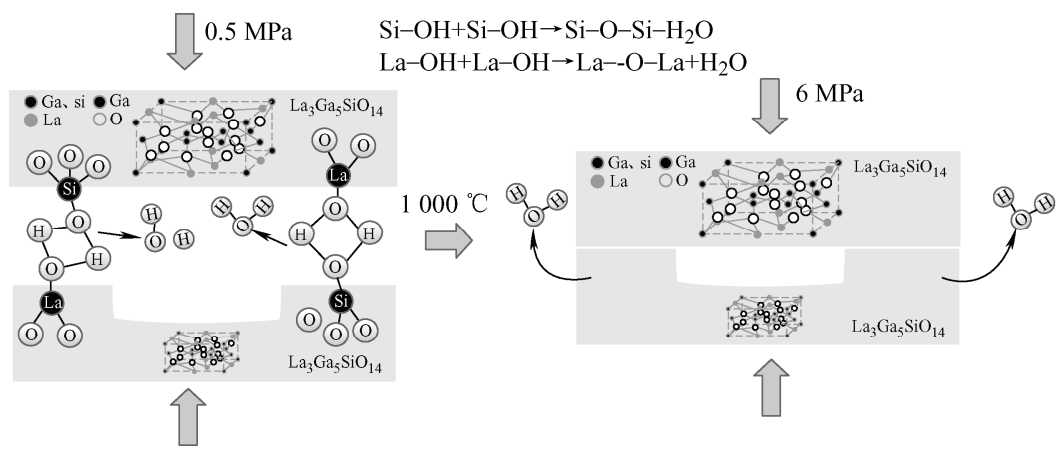

图 2 热压键合原子扩散图 


\section{2 高温压力器件微腔加工工艺}

\section{1 湿法刻蚀工艺}

由于硅酸镓镧单晶材料能够与各种酸碱溶液反 应, 因此对采用湿法刻蚀的方法在 LGS 晶片上实现 微腔结构增加了难度, 虽可实现表面微结构, 但除 形成微结构以外的晶体表面也会被酸或碱溶液腐 蚀。为解决此问题, 本文进行如下试验。试验采用 $10 \mathrm{~mm} \times 10 \mathrm{~mm} \times 0.5 \mathrm{~mm}$ 的双抛 $\mathrm{LGS}$ 晶片为基底。丙 酩、酒精清洗 LGS 基底表面杂质, 氮气吹干, 加热 台前烘; 在硅酸镓镧基底表面均匀旋涂负性光刻胶 (SU-8 2075), 设置匀胶机低转速 $500 \mathrm{r} / \mathrm{min}, 10 \mathrm{~s}$, 高 转速 $2500 \mathrm{r} / \mathrm{min}, 40 \mathrm{~s}$ 。软烘控制温度为 $65{ }^{\circ} \mathrm{C}-7 \mathrm{~min}$, $95{ }^{\circ} \mathrm{C}-37 \mathrm{~min}$; 紫外线光刻; 后烘温度 $65{ }^{\circ} \mathrm{C}-5 \mathrm{~min}$, $95{ }^{\circ} \mathrm{C}-15 \mathrm{~min}$, 随加热台自然冷却至室温; 使用 SU-8 2075 光刻胶专用显影液显影。将显影后的晶片用负 胶黏附在 PP 盒实现全包围的方式(除显影区域)以免 被酸腐蚀, 并进行烘烤。配置腐蚀溶液盐酸与磷酸 $1: 1,1: 2,1: 3$ 不同比例混合, 将预键合后的晶 片分别放入不同配比的腐蚀溶液, 由于腐蚀液有 强挥发性, 因此需用保鲜膜将其密封好, 水浴加 热到 $80{ }^{\circ} \mathrm{C}$ 进行腐蚀。前期课题组分析了不同配 比的磷酸与盐酸混合溶液在不同水浴温度下的刻 蚀速率, 分析结果可知, 水浴加热 $80{ }^{\circ} \mathrm{C}$ 时, 磷 酸与盐酸 $1: 1$ 混合的刻蚀速率最快, 刻蚀表面效 果最好 ${ }^{[34]}$ 。经过约 $2.5 \mathrm{~h}$ 的腐蚀, 得到腐蚀空腔。 刻蚀后的空腔及刻蚀流程图如图 3 所示。利用台阶 仪测试空腔深度约为 $100 \mu \mathrm{m}$, 直径为 $4 \mathrm{~mm}$ 。纵向 腐蚀速率约为 $42 \mu \mathrm{m} / \mathrm{h}$, 腐蚀速率与酸的浓度、体积 相关。

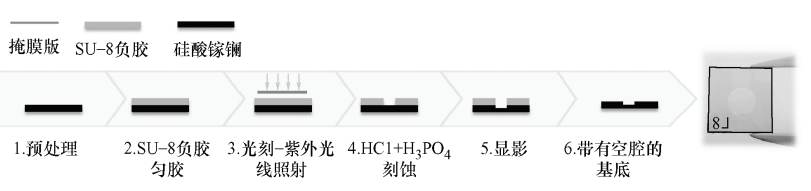

图 3 LGS 刻蚀流程示意图

\section{2 预键合加工工艺}

将 $10 \mathrm{~mm} \times 10 \mathrm{~mm} \times 0.5 \mathrm{~mm}$ 未刻蚀的双抛晶片与 带有空腔的晶片依次用食人鱼液(浓 $\mathrm{H}_{2} \mathrm{SO}_{4}$ : $\mathrm{H}_{2} \mathrm{O}_{2}=3: 1$ ) 在 $120{ }^{\circ} \mathrm{C}$ 加热台加热以去除附着在晶 片表面的有机物和金属杂质; 用标准清洗液 1 号液 (SC1)水浴加热以去除晶片表面的颗粒污染物; 再将 晶片放入食人鱼液中 $120{ }^{\circ} \mathrm{C}$ 下浸泡, 晶圆表面附着 亲水性的 $\mathrm{OH}^{-}$层, 最后用去离子水冲洗后使用氮气 吹干; 将清洗好的晶片键合面朝上放入培养血中,
使用 PVA Tepla 的 Ion Wave 10 等离子体系统进行氧 等离子体活化处理以增强键合面的表面活化能，设 置其参数为: 氧气流量 $200 \mathrm{sccm}$, 功率 $200 \mathrm{~W}$, 腔 室压强 $26.6 \mathrm{~Pa}$ (200 mTorr); 将活化后的晶片表面进 行湿法化学表面活化实现预键合。活化后的晶片表 面亲水性较好。亲水性由水接触角的大小反应, 水 接触角越小亲水性越好, 未经任何处理的样品的水 接触角较大, 其亲水性较差; 湿法清洗和氧等离子体 活化处理的样品相对仅经过氧等离子体处理的在活 化时间内水接触角变化较大，且仅经过氧等离子体处 理的在活化时间内水接触角小，因此选择经过氧等离 子体活化处理后的 LGS 晶片，其键合表面羟基含量 多，水接触角小，利于实现晶片之间的预键合。

\section{3 垫压键合加工工艺}

热压键合工艺是在高温条件下使 LGS 晶片表 面原子获得足够的能量，使表面原子在键合接触界 面处扩散, 形成共价键达到强力的键合 ${ }^{[30]}$ 。将预键 合之后的晶片置于真空单向热压烧结炉中进行高温 热压键合，试验所使用的真空单向热压烧结炉实物 图如图 4a 所示, 其真空极限为 $5 \times 10^{-3} \mathrm{~Pa}$, 真空单 向热压烧结炉的工作原理为经预键合处理后的晶片 放入石墨模具中，系统给电极加热使待键合的晶片 快速加热，通过液压洜和压头移动控制系统给石墨

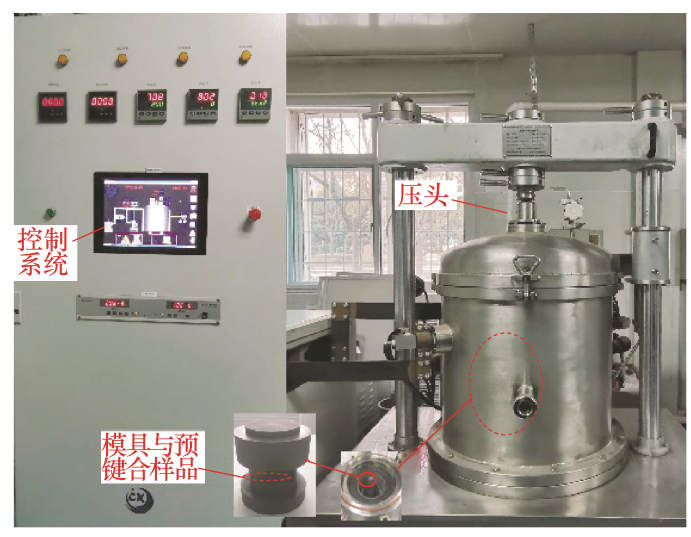

(a)

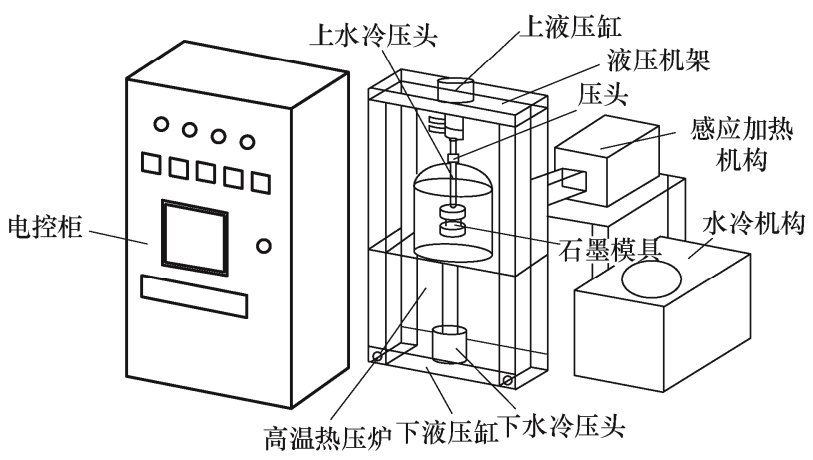

(b)

图 4 真空单向热压烧结炉 
模具施加压力, 最终实现晶片的键合, 工作原理图 如图 $4 \mathrm{~b}$ 所示。热压炉提供真空环境, 可使晶片直接 键合形成真空密封腔。

高温热压键合的温度选择范围一般是考虑在材 料熔点的 $50 \% \sim 70 \%$ 之间 ${ }^{[30]}$, 而且熔点的 $80 \%$ 为键 合的临界温度, 为探索 LGS 材料最佳的键合温度, 本文分别选择了 $900 \sim 1000{ }^{\circ} \mathrm{C}$ 的温度范围内进行 不同温度条件下键合试验。因为在键合温度小于 $900{ }^{\circ} \mathrm{C}$ 时, 晶片未能实现键合, 以及键合样品在高 于 $1000{ }^{\circ} \mathrm{C}$ 时, 虽实现两晶片的键合, 但晶片表面 出现裂痕, 以致未能形成密封空腔。前期课题组采 用拉伸试验机对键合后的晶片进行拉力测试, 以测 试晶片的键合强度, 测试结果表明在 $1000{ }^{\circ} \mathrm{C}$ 时, 键合强度最大, 拉应力为 $3.81 \mathrm{MPa}^{[35]}$ 。由此可得知 键合参数为 $1000{ }^{\circ} \mathrm{C}$ 下施加 $6 \mathrm{MPa}$ 的压力时晶片的 键合效果最佳。在最佳键合条件下键合过程为: 系统 预热从 $0{ }^{\circ} \mathrm{C}$ 上升至 $200{ }^{\circ} \mathrm{C}$, 保温 $0.5 \mathrm{~h}$, 以 $10{ }^{\circ} \mathrm{C} / \mathrm{min}$ 的速率升温至 $1000{ }^{\circ} \mathrm{C}$, 在 $1000{ }^{\circ} \mathrm{C}$ 的温度下保温 $2 \mathrm{~h}$, 同时施加 $6 \mathrm{MPa}$ 的压力。键合温度曲线如图 5 所示。经热压键合后得到密封压力腔。

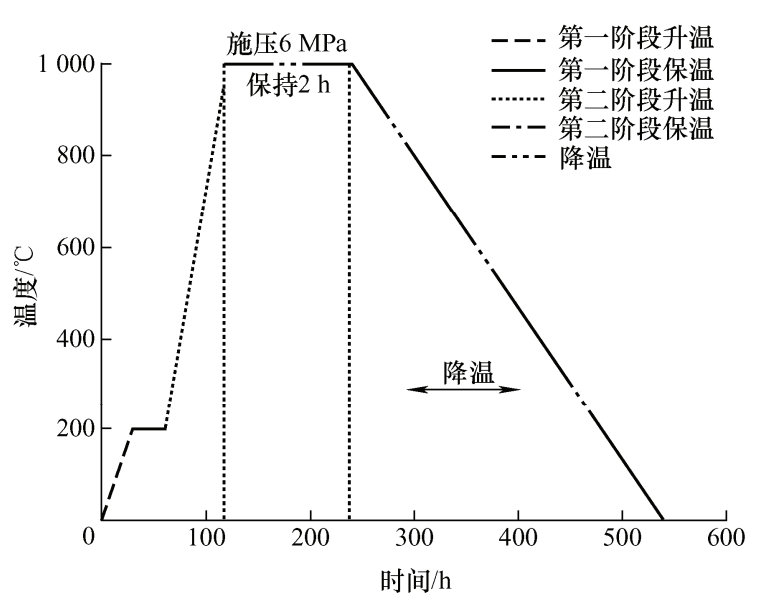

图 5 硅酸镓镧热压键合温度加热曲线

键合后的密封压力腔利用 HF-4 氦气检漏装置 和 NHJ-400 氦质谱检漏仪进行气密性检漏试验。对 气密腔进行氦质谱检漏试验, 氦泄漏率小于细检漏 率极限值; 气密腔在粗检中未出现连续冒泡现象, 表明腔体密封性良好, 满足制备高温压力传感器的 要求。

\section{3 高温压力器件制造及性能测试}

\section{1 高温压力器件原理及制造}

声表面波传感器由叉指换能器(IDT)、反射栅组
成。利用第 2 节制备好的带有压力密封空腔的 LGS 晶片作为制备声表面波高温压力器件的基底。在 LGS 基底上实现叉指结构试验的简单步骤如下:

(1) 将 LGS 基底使用丙酮+酒精清洗; (2) 在 LGS 基 底抛光表面旋涂正性光刻胶匀胶; (3) 显影; (4) 涂 有光刻胶表面溅射铂金属; (5) 剥离金属; (6) 在氮 气中退火处理, 以提高电极在高温环境中的工作稳 定性。本文制备的传感器叉指宽为 $4 \mu \mathrm{m}$, 波长 $\lambda$ 为 $16 \mu \mathrm{m}$, 孔径为 $100 \lambda$, 叉指对数为 80 对, 反射栅对 数 100 对, $\mathrm{LGS}$ 晶片的切向为 $\left(0^{\circ}, 138.5^{\circ}, 117^{\circ}\right)$, 复合电极为 $10 \mathrm{nmTi} / 150 \mathrm{nmPt} / 100 \mathrm{nmAlN}$ 。高温电 极防护层 AlN 是由激光脉冲沉积系统(Pulsed laser deposition, PLD)制备。压力传感器敏感结构图如 图 6 所示。声表面波压力传感原理为当外界环境压 力发生变化时, 位于压力谐振结构下方的空腔因受 力而发生形变, 压电材料的弹性系数、密度等物理 参数发生变化, 从而影响了声波的传播距离, 同时 也影响了声表面波的传播速度以及波长发生变化, 从而引起传感器的谐振频率发生变化。传感器的传 感特征用谐振频率来表示, 因此谐振频率的偏移就 可以表征压力的变化。通常情况下压力变化主要引 起声表面波传播速度的变化, 由 SAW 波速和温度 的关系 $f=\frac{v}{\lambda}$ 可以得到

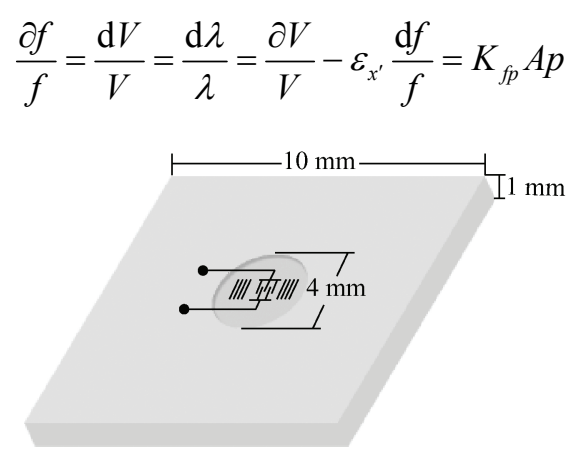

图 6 压力敏感结构图

利用扰动理论求出波速变化和应变 $\varepsilon_{x}$ 就可以得 到谐振频率偏移和压力变化的关系, $A$ 是归一化参 数, $K_{f p}$ 是压力灵敏度系数。

\section{2 微器件性能测试}

制备好的传感器如图 7a 所示。利用改造提升的 JT1500 高温-压力复合测试系统进行压力传感器的 高温压力复合测试, 测试系统主要由主控制系统、 温压控制系统、密封气压罐、水冷系统、真空系统 等组成, 其实物图如图 7b 所示。如图 7c、7d 所示 分别为压力传感器在常温和经 $1000{ }^{\circ} \mathrm{C}$ 高温下测试 的表面形貌图, 对比图 7c 与图 7d, 发现 $1000{ }^{\circ} \mathrm{C}$ 
高温下测试的叉指宽度较常温下测试的叉指宽度变 细, 但叉指条并未出现断裂, 表明传感器在经高温 加热后传感器仍保持良好性能。压力传感器的测试 结果如图 7e、7f 所示。

由测试结果图 7e 可得知, 声表面波压力传感器 在温度为 $500 \sim 1000{ }^{\circ} \mathrm{C}$ 的范围内, 压力从 $20 \mathrm{kPa}$ 变化到 $500 \mathrm{kPa}$, 其敏感膜片的形变引起压力传感器 的频率变化, 谐振频率随着压力的增大而降低, 具

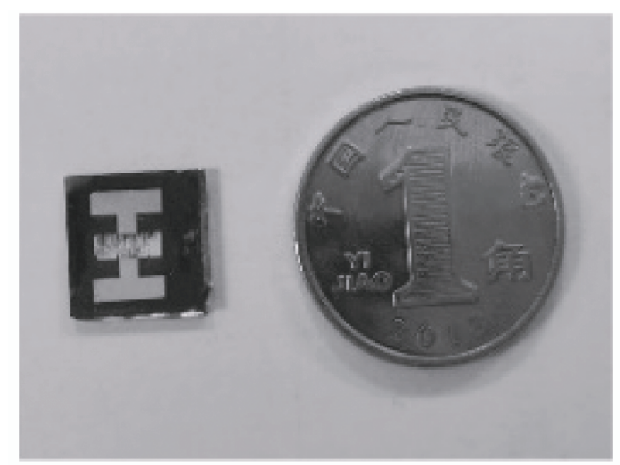

(a) 传感器实物图

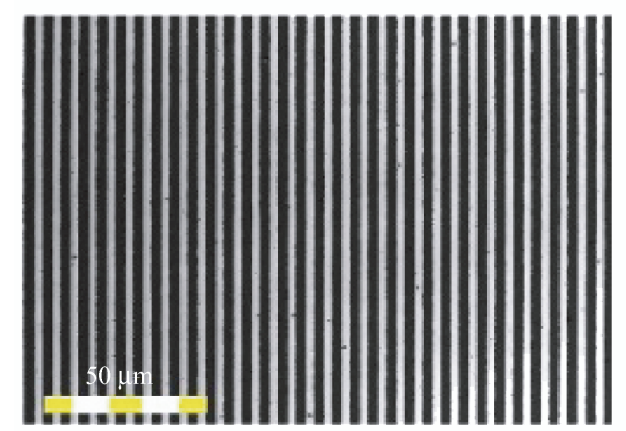

(c) 常温下传感器叉指电极表面形貌图

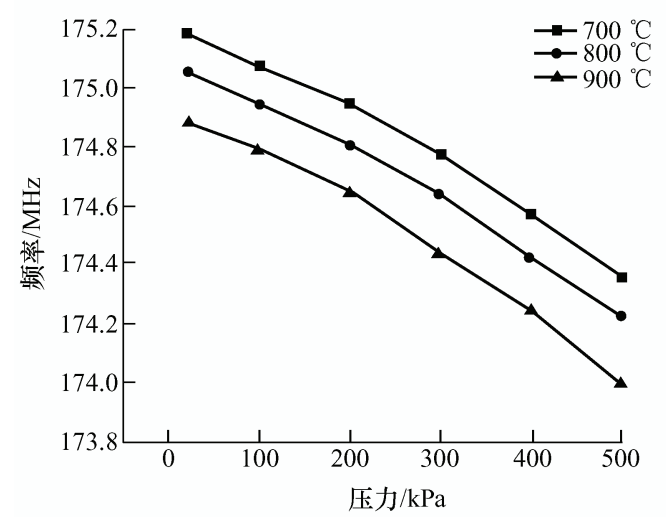

(e) $500 \sim 1000{ }^{\circ} \mathrm{C}$ 下在 $20 \sim 500 \mathrm{kPa}$ 压力 范围内测试结果
有良好的线性性, 可知压力传感器可在高温环境下 稳定工作。如图 7f 所示, 压力传感器在不同的压力 条件下, 频率随着温度的升高而降低, 谐振频率与 温度呈二次型关系。试验结果表明利用湿法刻蚀、 热压键合工艺制备的压力空腔完好, 验证了压力空 腔的微加工制备方法的可行性以及声表面波压力传 感器具有耐高温性能, 也为利用硬脆性材料制备带有 密封空腔的高温压力传感器提供了研究价值。

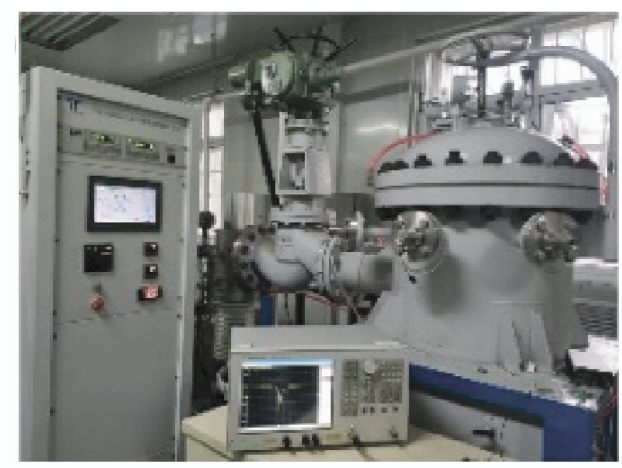

(b) JT1500高温-压力复合测试系统实物图

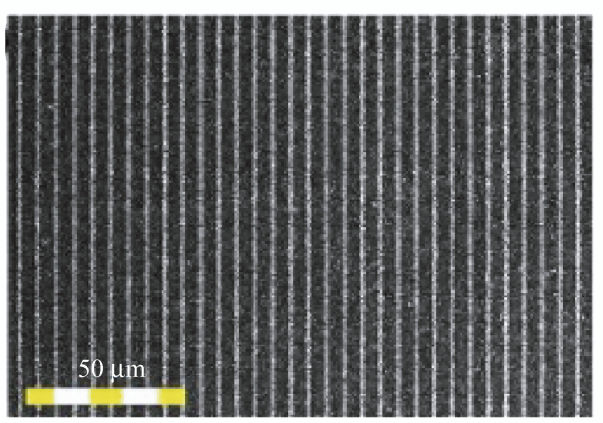

(d) $1000{ }^{\circ} \mathrm{C}$ 高温加热后传感器叉指电极表面形貌图

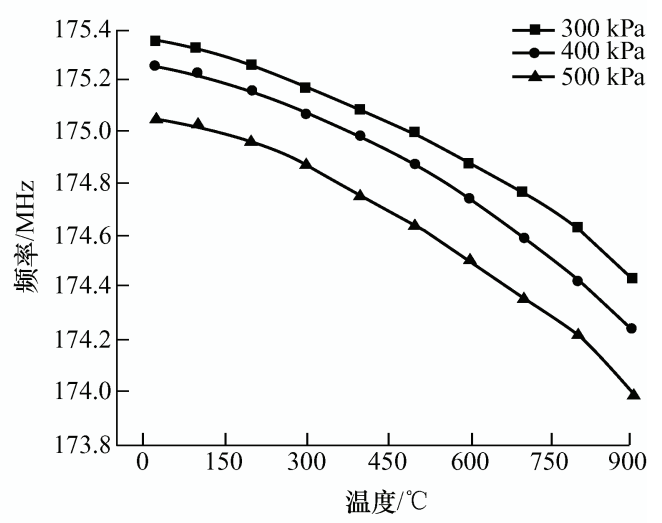

(f) 传感器在 $300 \sim 500 \mathrm{kPa}$ 的条件下的温度 与频率变化曲线图

图 7 性能测试实物及结果曲线

\section{4 结论}

本文针对硅酸镓镧硬脆性材料的难加工特性,
研究了硬脆性材料表面图形化的微加工制造技术, 根据材料的化学、力学特性, 实现了硬脆性材料表 面腔体微结构的制备。

(1) 常规的 LGS 晶片较厚 $(500 \mu \mathrm{m})$, 要形成较 
敏感的敏感膜片, 需要寻找较快的刻蚀速率的方法, 本文分析了硅酸镓镧压电材料的湿法刻蚀工艺机 理, 摸索了适合硅酸钾镧材料刻蚀的相关参数关系, 实现了 LGS 的微纳结构成形制造。

(2) LGS 晶片为硬脆性材料难加工, 常规的焊 接、粘接技术无法在超高温环境下稳定工作, 本文 根据材料的高温力学特性, 提出了高温热压直接键 合方法, 揭示了其微观机理, 探讨了键合面、力与 温度加载对键合影响的关系。试验采用氧等离子体 活化、湿法活化与高温退火结合的晶片键合的工艺, 分析不同温度加载与不同键合压力加载参数的影 响, 得出最佳键合条件: 键合温度 $1000{ }^{\circ} \mathrm{C}$, 表面持 续 $2 \mathrm{~h}$ 施加 $6 \mathrm{MPa}$ 压力, 实现 LGS 密封空腔的制造。 对键合后的密封空腔进行键合强度及气密性测试, 测 试表明键合后的密封空腔效果良好, 解决了硬脆性材 料制备高温压力传感器的绝压腔的难题, 为高温压力 传感器的制备提供了有效的方法与技术。

(3) 通过刻蚀、热压键合的 MEMS 微加工工艺 实现密封空腔制备, 并在制备好的密封空腔的敏感 膜片表面利用光刻、磁控溅射等工艺制备了基于 LGS 的 SAW 高温压力传感器。对 SAW 高温压力 传感器在 $20 \sim 500 \mathrm{kPa}, 25 \sim 1000{ }^{\circ} \mathrm{C}$ 的条件下进行 测试, 测试结果表明制备的压力传感器在 $25 \sim$ $1000{ }^{\circ} \mathrm{C}, 20 \sim 500 \mathrm{kPa}$ 范围内正常工作, 表明了基 于 LGS 的膜片式声表面波压力传感器可在高温下 稳定工作, 验证了该传感器微结构加工制造技术的 可行性。

\section{参 考 文 献}

[1] 王静, 杨杰, 赵文斌. 航天飞行器外防热复合材料发展 概况[J]. 材料导报， 2018，32(S2): 425-429.

WANG Jing, YANG Jie, ZHAO Wenbin. Development of thermal insulation composite materials for space vehicles[J]. Material Review, 2018，32(S2): 425-429.

[2] 邵靖, 段力, 王强, 等. MEMS 高温温度传感器的研制 与测量精度研究 [J]. 传感技术学报, 2017, 30(9): 1352-1358.

SHAO Jing, DUAN Li, WANG Qiang, et al. Development and measurement accuracy of MEMS high temperature sensor[J]. Journal of Sensing Technology, 2017, 30(9): 1352-1358.

[3] DUAN F L, DING Guifu, GAO Junchao, et al. High temperature sensors for intelligent aero-engine applications[C/CD]//33rd AIAA Aerodynamic Measurement
Technology and Ground Testing Conference, 2017.

[4] MENON D B. Fatigue life reliability based on measured usage, flight loads, and fatigue strength variations[J]. J. Am. Helicopter. Soc., 2011, 56(3): 1-18.

[5] JOHNSON R W, EVANS J L, JACOBSEN P, et al. The changing automotive environment: High-temperature electronics[J]. IEEE Transactions on Electronics Packaging Manufacturing, 2005, 27(3): 164-176.

[6] 康占祥, 黄漫国, 戴嫣青, 等. 新型航空发动机测试传 感器的发展趋势 [J]. 测控技术, 2012, 31(12): 1-3. KANG Zhanxiang, HUANG Manguo, DAI Yanqing, et al. Development trend of new aero-engine test sensor[J]. Measurement and Control Technology, 2012, 31(12): 1-3.

[7] 王尧, 张国华. 模拟电子技术 $[\mathrm{M}]$. 南京: 东南大学出 版社, 1994.

WANG Yao, ZHANG Guohua. Analog electronics[M]. Nanjing: Southeast University Press, 1994.

[8] JATLAOUI MM, PONS P, AUBERT H. Radio frequency pressure transducer[C]//Microwave Conference, 2007, European. IEEE, 2007: 736-739.

[9] JATLAOUI M M, PONS P, AUBERT H. Pressure micro-sensor based on radio-frequency transducer[C]// Microwave Symposium Digest, 2008 IEEE MTT-S International. IEEE, 2008: 1203-1206.

[10] JATLAOUI M, CHEBILA F, PONS P, et al. Pressure sensing approach based on electromagnetic transduction principle[C]// Microwave Conference, 2008. APMC 2008. Asia-Pacific. IEEE, 2008: 1-4.

[11] SCOTT S, PEROULIS D. A capacitively-loaded MEMS slot element for wireless temperature sensing of up to $300{ }^{\circ} \mathrm{C}[\mathrm{C}] / /$ Microwave Symposium Digest, 2009. MTT'09. IEEE MTT-S International. IEEE, 2009 : 1161-1164.

[12] JATLAOUI M, CHEBILA F, PONS P, et al. New micro-sensors identification techniques based on reconfigurable multi-band scatterers[C]/Microwave Conference, 2009. APMC 2009. Asia Pacific. IEEE, 2009: 968-971.

[13] BLAZQUEZ G, PONS P, BOUKABACHE A. Capabilities and limits of silicon pressure sensors[J]. Sensors \& Actuators, 1989, 17(3): 387-403.

[14] REN J, WARD M, KINNELL P, et al. Plastic 
deformation of micromachined silicon diaphragms with a sealed cavity at high temperatures $[\mathrm{J}]$. Sensors, 2016, 16(2): 204.

[15] 李丹丹, 梁庭, 李赛男, 等. 基于 MEMS 工艺的 SOI 高温压力传感器设计 [J]. 传感技术学报, 2015, 28(9): 1315-1320.

LI Dandan, LIANG Ting, LI Sainan, et al. Design of SOI high temperature pressure sensor based on MEMS process[J]. Journal of Sensing Technology, 2015，28(9): $1315-1320$

[16] CHANDER S, SINHA S K, KUMAR S, et al. Temperature analysis of $\mathrm{Ge} / \mathrm{Si}$ heterojunction SOI-Tunnel FET[J]. Superlattices \& Microstructures, 2017, 110: $162-170$.

[17] 巩鹏亮. 深亚微米全耗 SOI CMOS 的高温应用分析 [D]. 西安: 西安理工大学, 2008.

GONG Pengliang. High temperature application analysis of deep submicron total consumption SOI CMOS[J]. Xi'an: Xi'an University of Technology, 2008.

[18] GUO S, ERIKSEN H, CHILDRESS K, et al. High temperature smart-cut SOI pressure sensor[J]. Sensors and Actuators: A Physical, 2009, 154(2): 255-260.

[19] LUKCO D, SAVRUN E, OKOJIE R S, et al. Demonstration of $\mathrm{SiC}$ pressure sensors at $750{ }^{\circ} \mathrm{C}[\mathrm{R}]$. NASA Report U1522, 2014.

[20] SHAO Shiqian, LIEN Weicheng, MARALANI A, et al. 4H-Silicon carbide $\mathrm{p}-\mathrm{n}$ diode for high temperature $\left(600{ }^{\circ} \mathrm{C}\right)$ environment applications[J]. Materials Science Forum, 2015, 821-823: 636-639.

[21] POTBHARE S , GOLDSMAN N , LELIS A , et al. A physical model of high temperature $4 \mathrm{H}-\mathrm{SiC}$ MOSFETs[J]. IEEE Transactions on Electron Devices, 2008, 55(8): 2029-2040.

[22] DEVKOTA J, GREVE D W, HONG T, et al. An 860 $\mathrm{MHz}$ wireless surface acoustic wave sensor with a metal-organic framework sensing layer for $\mathrm{CO}_{2}$ and $\mathrm{CH}_{4}[\mathrm{~J}]$. IEEE Sensors Journal, 2020, 20(17): 9740-9747.

[23] JAGANNATH D, PAUL O, DAVID G. SAW sensors for chemical vapors and gases[J]. Sensors, 2017, 17(4): 801-828.

[24] TAO Han, JI Xiaojun, SHI Wenkang. Optimal pressure-sensitive cuts for surface acoustic waves on langasite $[\mathrm{J}]$. Science in China Series F: Information
Sciences, 2006, 49: 254-261.

[25] TAZIEW R M. Stress, temperature and pressure behavior of SAW on langasite plates[C/CD]//IEEE International Frequency Control Symposium \& Pda Exhibition. IEEE, 2001.

[26] SHU Lin, PENG Bin, CUI Yilin, et al. Effects of AlN coating layer on high temperature characteristics of Langasite SAW sensors[J]. Sensors， 2016， 16(9): 1436.

[27] BARDONG J, AUBERT T, NAUMENKO N, et al. Experimental and theoretical investigations of some useful Langasite cuts for high-temperature SAW applications[J]. IEEE Transactions on Ultrasonics Ferroelectrics \& Frequency Control, 2013, 60(4) : 814-823.

[28] SAKHAROV S, KONDRATIEV S, ZABELIN A, et al. Theoretical and experimental investigation of langasite as material for wireless high temperature SAW sensors[C]// Ultrasonics Symposium, IEEE, 2010: 535-538.

[29] 黄海. 采用 in-film 结构刻蚀阻挡层进行双镶嵌刻蚀工 艺的研究[D]. 上海: 复旦大学, 2012 .

HUANG Hai. The in-film structure etching barrier layer was used to study the double mosaic etching process[D]. Shanghai: Fudan University, 2012.

[30] 阮传植. 多层硅硅直接键合实验研究[D]. 武汉: 华中科 技大学, 2009 .

RUAN Chuanzhi. Experimental study on direct bonding of multilayer silicon[D]. Wuhan: Huazhong University of Technology, 2009.

[31] 周文和. 快速响应湿敏元件特性及其测试方法的研究 [D]. 兰州: 兰州交通大学, 2014 .

ZHOU Wenhe. Study on the characteristics of rapid respo-nse humidity sensor and its testing method[D]. Lanzhou: Lanzhou Jiaotong University, 2014.

[32] 李旺旺. 蓝宝石高温压力传感器关键技术研究 $[D]$. 太 原：中北大学，2019.

LI Wangwang. Research on key technology of sapphire high temperature pressure sensor[D]. Taiyuan: North University of China, 2019

[33] 间忠龙. 基于激光制冷的四波混频和光学微腔关键技 术研究[D]. 成都：电子科技大学, 2014 .

YAN Zhonglong. Research on key technologies of four-wave mixing and optical microcavity based on laser cooling[D]. Chengdu: University of Electronic Science 
and Technology, 2014.

[34] XU Fangmeng, TAN Qiulin. Langasite micromachining technology applied to surface acoustic wave sensors in ultra-high temperatures $[\mathrm{C} / \mathrm{CD}] / /$ The 34 th $\quad$ IEEE International Conference on Micro-electro-mechanical Systems.

[35] XU Fangmeng, XUE Tao, LIANG Xiaorui, et al. High-temperature direct bonding of langasite using oxygen plasma activation[J]. Scripta Materialia, 2021, 194(4): 113681.

作者简介: 梁晓瑞, 女, 1993 年出生, 博士研究生。主要研究方向为高 温恶劣环境下微纳器件。

E-mail: B1906058@st.nuc.edu.cn

谭秋林(通信作者), 男, 1979 年出生, 博士, 教授, 博士研究生导师。 主要研究方向为特种微传感器与集成系统。

E-mail: tanqiulin@nuc.edu.cn 